\title{
The Metabolic Effects of Traditional Chinese Medication Qiliqiangxin on H9C2 Cardiomyocytes
}

\author{
Shenghui Lin ${ }^{a}$ Xiaoting Wu Lichan Tao $^{a}$ Yihua Beib,c Haifeng Zhang ${ }^{\mathrm{a}}$ \\ Yanli Zhou ${ }^{a}$ Shutong Shen ${ }^{\mathrm{a}}$ Junjie Xiao ${ }^{\mathrm{b}, \mathrm{c}}$ Xinli Lia \\ aDepartment of Cardiology, The First Affiliated Hospital of Nanjing Medical University, Nanjing, \\ ${ }^{b}$ Regeneration and Ageing Lab, Experimental Center of Life Sciences, School of Life Science, Shanghai \\ University, Shanghai, 'Shanghai Key Laboratory of Bio-Energy Crops, School of Life Sciences, Shanghai \\ University, Shanghai, China
}

\section{Key Words}

Qiliqiangxin (QLQX) • Cardiomyocytes • Mitochondria • PGC-1 $\alpha$

\begin{abstract}
Background/Aims: A traditional Chinese medicine, Qiliqiangxin (QLQX) has been identified to perform protective effects on myocardium energy metabolism in mice with acute myocardial infarction, though the effects of QLQX on myocardial mitochondrial biogenesis under physiological condition is still largely elusive. Methods: H9C2 cells were treated with different concentrations of QLQX $(0.25,0.5$, and $1.0 \mu \mathrm{g} / \mathrm{mL})$ from 6 to 48 hours. Oxidative metabolism and glycolysis were measured by oxygen consumption and extracellular acidification with XF96 analyzer (SeaHorse). Mitochondrial content and ultrastructure were assessed by Mitotracker staining, confocal microscopy, flow cytometry, and transmission electron microscopy. Mitochondrial biogenesis-related genes were measured by qRT-PCR and Western blot. Results: H9C2 cells treated with QLQX exhibited increased glycolysis at earlier time points $(6,12$, and 24 hours), while QLQX could enhance oxidative metabolism and mitochondrial uncoupling in $\mathrm{H} 9 \mathrm{C} 2$ cells with longer duration of treatment (48 hours). QLQX also increased mitochondrial content and mitochondrial biogenesis-related gene expression levels, including 16sRNA, SSBP1, TWINKLE, TOP1MT and PLOG, with an activation of peroxisome proliferator-activated receptor coactivator 1 alpha (PGC-1 $\alpha$ ) and its downstream effectors. Silencing PGC- $1 \alpha$ could abolish the increased mitochondrial content in $\mathrm{H} 9 \mathrm{C} 2$ cells treated with QLQX. Conclusion: Our study is the first to document enhanced metabolism in cardiomyocytes treated with QLQX, which is linked to increased mitochondrial content and mitochondrial biogenesis via activation of PGC-1 $\alpha$.




\section{Introduction}

Qiliqiangxin (QLQX), a traditional Chinese medicine, has previously been reported to perform protective effects in 512 heart failure patients in a multicenter randomized doubleblind study [1]. Also, QLQX treatment was shown to reduce myocardial apoptosis and cardiac fibrosis, thus ameliorating cardiac remodeling and improving cardiac function in a murine model of acute myocardial infarction (AMI) [2]. As we know, PPARs are a group of nuclear receptor proteins which play essential roles in the regulation of carbohydrate, lipid, and protein metabolism. Among PPARs family, PPAR coactivator-1 $\alpha$ (PGC-1 $\alpha$ ), was identified as a master transcriptional co-regulator for mitochondrial biogenesis. Thus, the regulatory effect of QLQX on PGC-1 $\alpha$ may provide a chance for QLQX as a potential intervention on mitochondrial dysfunction. Besides that, another study demonstrated that QLQX significantly prevented cardiac hypertrophy induced by ascending aorta constriction (TAC) in rat, which was linked to the reduced accumulation of free fatty acids and lactic acid, as well as to the improved mitochondrial function via activation of 5'AMP-activated protein kinase (AMPK)/ PGC- $1 \alpha$ axis [3]. Recently, another study reported that chronic administration of QLQX improved endothelium-dependent relaxation (EDR) and endothelial integrity by balancing endothelial nitric oxide synthase (eNOS) and inducible nitric oxide synthase (iNOS) activity in diabetic rat aortas, further confirming the therapeutic role of QLQX on cardiac energy metabolism [4]. Although the protective regulation of QLQX on injured mitochondria has achieved significant advance, the effect of QLQX on physiological mitochondrial function is still largely elusive.

Mitochondria are essential and key regulators of cellular bioenergetics [5]. The human heart consumes a lot of ATP daily to support basic energy metabolism and persistent pump function, while mitochondrial oxidative phosphorylation (OXPHOS) occupies the majority of high demand for ATP (>95\%), and actually about $40 \%$ of the cytoplasmic space in adult cardiac myocyte is occupied by mitochondria [6]. Importantly, cardiomyocyte mitochondria could respond to changes in energy substrate availability, workload, or energy demands in injured myocardium, thus establishing a close contact between mitochondria and cardiac homeostasis [7-9]. Mitochondrial dysfunction was demonstrated to contribute to cardiomyopathy with various clinical manifestations, and impaired mitochondrial homeostasis was also reported to lead to heart failure over time, for example, the ageing heart [10]. Consequently, exploring new therapeutic strategy to improve and stabilize mitochondrial function is of great clinical significance.

Mitochondrial biogenesis is regulated by a complex regulatory network. PGC- $1 \alpha$ is a key regulator participating in mitochondrial biogenesis and oxidative metabolism in the heart [11]. Besides that, PGC-1 $\alpha$ also induces mitochondrial replication by increasing the expression levels of mitochondria-related genes, such as mitochondrial transcription factor A (TFAM), single strand binding protein (SSBP) and transcription factor B2 (TFB2M) $[12,13]$. Several reports have demonstrated that mitochondrial function is increased by activating PGC-1 $\alpha[14,15]$. PGC-1 co-activators are also identified as inducible enhancers for transcriptional regulators participating in mitochondrial biogenesis, which include estrogen-related receptor (ERRs), nuclear respiratory factor-1 (NRF-1) and PPARs [16, 17], among which NRF-1 upregulates almost all the nuclear mitochondrial gene expression levels [18].

In this study, we investigated the regulatory effect of QLQX on the mitochondrial function during physiological condition using rat $\mathrm{H} 9 \mathrm{C} 2$ cardiomyocyte cell line, particularly focusing on oxygen consumption rate (OCR) and extracellular acidification (ECAR). Our results may provide a novel therapeutic strategy for improving and stabilizing mitochondrial function by using QLQX with PGC- $1 \alpha$ activation. 


\section{Cellular Physiology Cell Physiol Biochem 2015;37:2246-2256

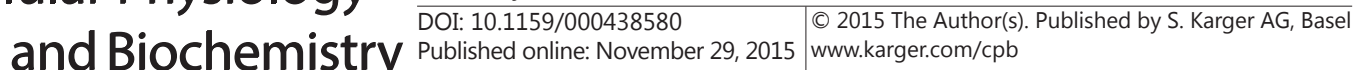 \\ Lin et al.: Metabolic Effects of Qiliqiangxin on Cardiomyocytes}

\section{Materials and Methods}

\section{Cell culture and treatment}

Rat cardiomyocyte H9C2 cell line was purchased from Shanghai Academy of Science (Shanghai, China) and were cultured in Dulbecco's modified eagle's medium (DMEM) with $4500 \mathrm{mg} / \mathrm{L}$ glucose and supplemented with $10 \%$ fetal bovine serum (FBS) and $100 \mathrm{U} / \mathrm{mL}$ penicillin/streptomycin in humidified air $\left(5 \% \mathrm{CO}_{2}\right)$ at $37^{\circ} \mathrm{C}$. QLQX, provided by Shijiazhuang Yiling Pharmaceutical Co. Ltd (Shijiazhuang, Hebei, China), was diluted in culture media to various concentrations $(0.25,0.5$, and $1.0 \mu \mathrm{g} / \mathrm{mL})$. Cells were treated with QLQX for either short duration (6,12, and 24 hours) or long duration (48 hours). Additionally, to examine the functional role of PGC- $1 \alpha$ in QLQX-induced changement in cardiomyocyte metabolism, cells were transfected with either PGC- $1 \alpha$ siRNA (75 nM) or negative control siRNA for 48 hours using lipofectamine 2000.

\section{Metabolic assay}

Cellular metabolism is a process containing substrate uptake (oxygen, glucose and fatty acid) and energy transition through several enzymatically controlled oxidation or reduction reactions. H9C2 cells were plated at a density of $0.5 \times 10^{5}$ cells /well in 96-well microtiter plates. After 24 hours, cells were treated with different dose of QLQX $(0.25,0.5$, and $1.0 \mu \mathrm{g} / \mathrm{mL})$ and incubated for 6 to 48 hours. Following treatment, culture media was exchanged and replaced with $100 \mu \mathrm{L}$ XF Assay Medium-modified DMEM containing 4500 $\mathrm{mg} / \mathrm{L}$ glucose (Seahorse Bioscience, Billerica, MA, USA), and then incubated at $37^{\circ} \mathrm{C}$ without $\mathrm{CO}_{2}$ for 1 hour. Extracellular acidification (ECAR), an indirect marker of glycolytic capacity, and oxygen consumption rate (OCR), a measure of oxidative metabolism, were detected by using SeaHorse XF96 Extracellular Analyzer and XF96 Software (Seahorse Bioscience, Billerica, MA, USA) according to manufacturers' protocols. The detection of cellular metabolism was repeated at least three times.

\section{Mitotracker staining and confocal microscopy}

H9C2 cells were plated in chamber-slides (BD Bioscience, Sparks, MD, USA) at a density of $1 \times 10^{6}$ cells/ well and treated with or without QLQX for 48 hours. Cells were then stained with Mitotracker (Beyotime Biotechnology, Nantong, Jiangsu, China) at a concentration of $200 \mathrm{nM}$ diluted in pre-warmed culture media for 45 minutes. After that cells were fixed in $4 \%$ formaldehyde in pre-warmed culture media and stained with DAPI (Invitrogen, Carlsbad, CA, USA) to marker cell nuclei. A total of 20 fields/well (400 x magnification) were taken by confocal microscope (Carl Zeiss, Thuringia, Germany). Cell size and fluorescence intensity were measured by Image J and Zeiss software, respectively.

\section{Mitotracker staining and flow cytometry}

H9C2 cells were seeded in 6-well plates at a density of $1 \times 10^{6}$ cells/well. After 24 hours, cells were treated with or without QLQX for 48 hours. Following treatment, cells were stained with Mitotracker (Beyotime Biotechnology, Nantong, Jiangsu, China) at a concentration of $200 \mathrm{nM}$ for 45 minutes, and then the media containing Mitotracker was removed. After, cells were washed with DPBS three times and peptized by trypsin without Ethylene Diamine Tetraacetie Acid (EDTA) and ultimately suspended in pre-warmed DPBS. Group mean fluorescence was measured by Facscalibur filtering $488 \mathrm{~nm}$.

\section{Transmission electron microscopy}

To evaluate the effect of QLQX on cell ultrastructure, especially mitochondria, transmission electron microscopy was conducted after cells were treated with or without QLQX. Following treatment as previously described, $\mathrm{H} 9 \mathrm{C} 2$ cells were fixed in $2.5 \%$ glutaraldehyde for 1 hour and then treated with $1 \%$ osmium tetroxide. After, cells were dehydrated and embedded in durcupan. Every sample was cut into $60 \mathrm{~nm}$ by using a diamond knife and mounted on Cu-grids, images were viewed by transmission electron microscope at $6000 \mathrm{x}$ magnification for analysis. All measurements were carried out within the same software package.

Reverse transcription polymerase chain reaction (RT-PCR) and Real-Time PCR

To analyze metabolic related mRNA expression levels, total RNA of H9C2 cells was extracted by using miRNeasy Mini Kit (Qiagen, Hilden, Germany) following manufacturer's protocols. Total RNA (400 ng) was reverse transcribed using Bio-Rad iScripTM cDNA Synthesis Kit (Bio-Rad, Hercules, CA, USA) in a volume 


\section{Cellular Physiology Cell Physiol Biochem 2015;37:2246-2256

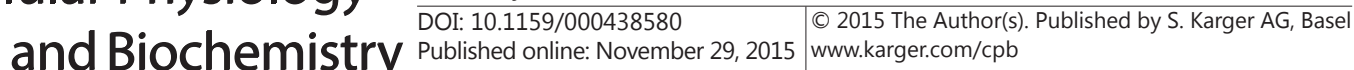 \\ Lin et al.: Metabolic Effects of Qiliqiangxin on Cardiomyocytes}

Table 1. Primers used in this study

\begin{tabular}{llll}
\hline Gene & Species & Forward & Reverse \\
\hline PGC-1 $\alpha$ & rat & GGAGCAATAAAGCAAAGAGCA & GTGTGAGGAGGGTCATCGTT \\
NRF1 & rat & TTGATGGACACTTGGGTAGC & GCCAGAAGGACTGAAAGCAG \\
TFAM & rat & CAGAGTTGTCATTGGGATTGG & TTCAGTGGGCAGAAGTCCAT \\
GLUT4 & rat & GTATGTTGCGGATGCTATGG & CCTCTGGTTTCAGGCACTCT \\
UCP3 & rat & CACGGATGTGGTGAAGGTC & CTGGCGATGGTTCTGTAGG \\
NRF2B & rat & GCCGTTTAGTCTCCGTGAAC & TCGTACTCCCAAGGCTGTGT \\
NRF2A & rat & AGCAGAAGCACATCTCGTTG & CCCATCTCGTCACTTGCTCT \\
TFB1M & rat & AAGGAAGTGGCGGAGAGAC & GGGATTGTAAAGAGGTGCTC \\
TFB2M & rat & TGCGGATGGAGAGTTACAAG & ACACCTGCTGACCAAGGAAC \\
PLOG & rat & CTCCTACCTGCCTGTCAACC & GCTCCATCAGCGACTTCTTC \\
TOP1MT & rat & CCAAGGTGTTTCGGACCTAC & GTTTGCCCGGTTGTAAGCTA \\
SSBP1 & rat & AGCCAGCAGTTTGGTTCTTG & ATCGCCACATCTCATTTGTT \\
TWINKLE & rat & GAGGACAGGGAGGAGGTCTT & TGGTAAGGCCAAACATCACA \\
16sRNA & rat & GACCCTGCTTGTAGCTGACC & ACCCTGATCCTTGAGACTGG \\
TBP2 & rat & TGTGAATACTGGTGCTGAG & GGCATGAGACAAGACCTATA \\
\hline
\end{tabular}

of $10 \mu \mathrm{L}$ system. The forward and reverse primers were shown in Table 1. Real-time PCR was performed using Bio-Rad SYBR qPCR (Bio-Rad, Hercules, CA, USA) in the Applied Biosystems Prism 7900HT system. The cDNA was amplified through 40 cycles of $15 \mathrm{~s}$ at $95^{\circ} \mathrm{C}, 30 \mathrm{~s}$ at $60^{\circ} \mathrm{C}$, and $30 \mathrm{~s}$ at $72^{\circ} \mathrm{C}$ for each gene. TBP2 was used for the internal control.

Immunoblotting analysis for $P G C-1 \alpha$

H9C2 cells were seeded in $6 \mathrm{~cm}$ dishes and treated with or without QLQX at a concentration of 0.5 $\mu \mathrm{g} / \mathrm{mL}$ for 48 hours, and then cells were lysed using RIPA buffer (Beyotime Institute of Biotechnology) containing a protease inhibitor mix (Sigma, St.louis, MO,USA) followed by incubation for 45 minutes on ice. Bicinchoninic acid protein assay kit (BCA, Thermo fisher, Waltham, MA, USA) was used to measure protein concentrations. Total protein (30 $\mathrm{gg} / \mathrm{sample}$ ) was separated in SDS-PAGE and electro-transferred to PVDF membranes. After blocking in TBST-5\% non-fat milk powder for 1 hour, membranes were incubated at $4^{\circ} \mathrm{C}$ for 14 hours in PGC-1 $\alpha$ (1:1000 dilution; NOVUS, Littleton, COLO, USA) primary antibody. $\beta$-actin (1:1000 dilution; Cell Signaling Technology, Boston, Massachusetts, USA) was used as loading control. All proteins were detected by chemiluminescence using the ECL Plus Western Blotting Detection Kit (Thermo fisher, Waltham, MA, USA) with Image lab software (Bio-Rad, Hercules, CA, USA). The quantification of PGC-1 $\alpha$ was normalized to $\beta$-actin by using Image lab software (Bio-Rad, Hercules, CA, USA).

Statistical analysis

Data were presented as mean \pm SE. Student's $t$-test, Chi-squares test or one-way ANOVA with Bonferroni's post-hoc test was used to compare values between groups. $P$ value $<0.05$ was considered significant. All analyses were performed using GraphPad Prism 5 software.

\section{Results}

QLQX enhances cardiomyocyte metabolism

To investigate the effects of QLQX on H9C2 cell metabolism, we treated cells with incremental concentrations $(0.25,0.5$, and $1.0 \mu \mathrm{g} / \mathrm{mL})$ and durations $(6,12,24$, and 48 hours) of QLQX. QLQX from $0.5 \mu \mathrm{g} / \mathrm{mL}$ increased basal oxygen consumption rate (OCR) following 48 hour treatment (Fig. 1A). However, $0.5 \mu \mathrm{g} / \mathrm{mL}$ of QLQX did not elevate basal OCR at earlier time points $(6,12$, and 24 hours) (Fig. 1B). Peak oxidative metabolism, which refers to chemically induced peak oxidative capacity, shared a similar change with basal OCR. QLQX significantly elevated peak OCR by $27.0 \%$ at $0.5 \mu \mathrm{g} / \mathrm{mL}$ after 48 hour treatment (Fig. 
A.

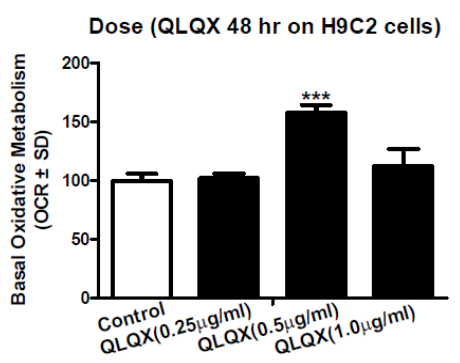

C.

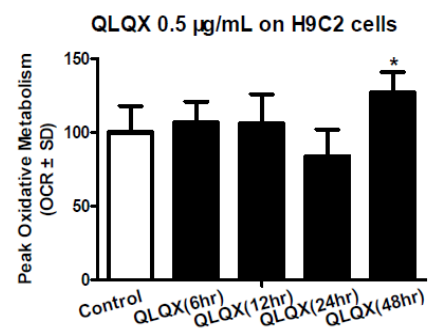

B.

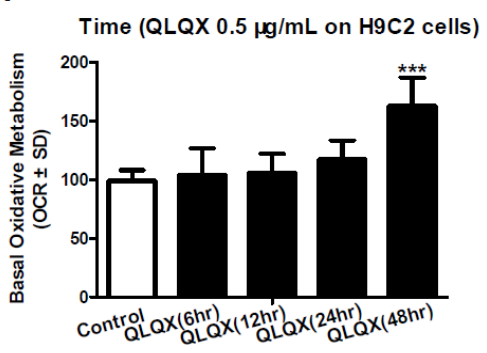

D.

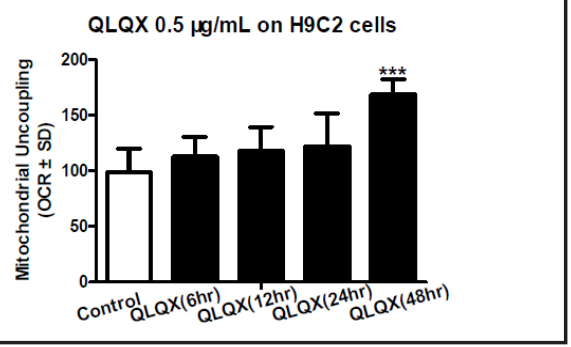

Fig. 1. QLQX enhances cardiomyocyte oxidative metabolism. (A) Basal oxygen consumption rate (OCR) of H9C2 cells treated with QLQX at different concentrations $(0.25,0.5$, and $1.0 \mu \mathrm{g} / \mathrm{mL})$ for 48 hours $(\mathrm{n}=8)$. (B) Basal OCR of H9C2 cells treated with $0.5 \mu \mathrm{g} / \mathrm{mL}$ of QLQX for different durations $(6,12,24$, and 48 hours) (n = 8). (C) Peak OCR of H9C2 cells treated with $0.5 \mu \mathrm{g} / \mathrm{mL}$ of QLQX for 6 to 48 hours ( $\mathrm{n}=8$ ). (D) Mitochondrial uncoupling (proton leak) of H9C2 cells treated with $0.5 \mu \mathrm{g} / \mathrm{mL}$ of QLQX for 6 to 48 hours $(\mathrm{n}=8){ }^{*}, P<0.05$; ***, $P<0.001$.

Fig. 2. The effect of QLQX on cardiomyocyte glycolytic metabolism. (A) Basal glycolytic metabolism indicated by extracellular acidification rate (ECAR) of H9C2 cells treated with $0.5 \mu \mathrm{g} / \mathrm{mL}$ of QLQX for 6 to 48 hours (n = 8). (B) Peak glycolytic metabolism of $\mathrm{H} 9 \mathrm{C} 2$ cells treated with $0.5 \mu \mathrm{g} / \mathrm{mL}$ of QLQX for 6 to 48 hours (n = 8). (C) Metabolic reliance, indicated as a ratio of basal oxidative metabolism (oxygen consumption rate, OCR) to glycolytic metabolism (extracellular acidification rate, ECAR) following QLQX treatment as described above $(\mathrm{n}=8)$. $*, P<0.05$; ** $P<0.01$; ***, $P<0.001$.
A.

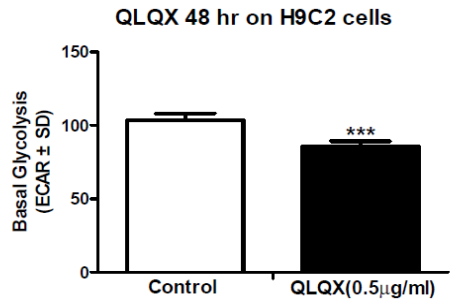

QLQX $0.5 \mu \mathrm{g} / \mathrm{mL}$ on $\mathrm{H} 9 \mathrm{C} 2$ cells

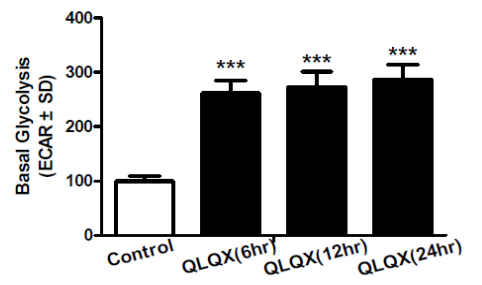

B.

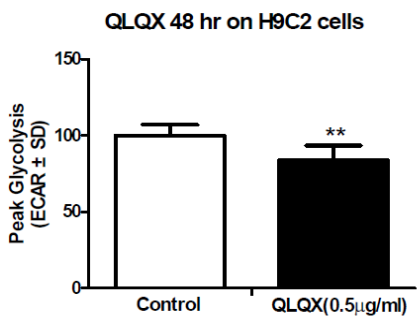

QLQX $0.5 \mu \mathrm{g} / \mathrm{mL}$ on $\mathrm{H} 9 \mathrm{C2}$ cells

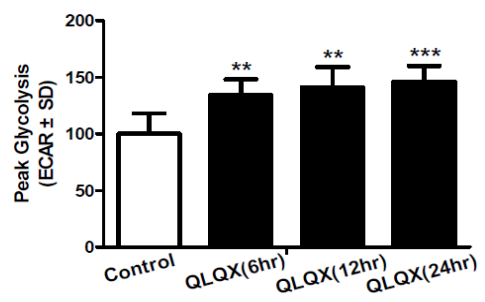

C.

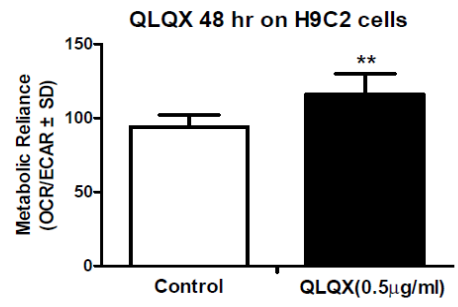

QLQX $0.5 \mu \mathrm{g} / \mathrm{mL}$ on $\mathrm{H} 9 \mathrm{C} 2$ cells

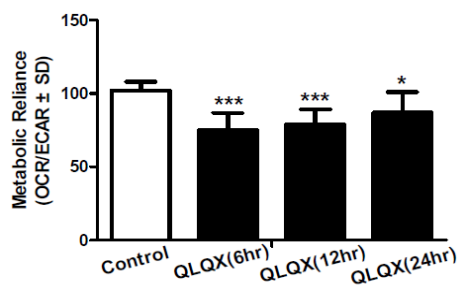


Fig. 3. QLQX increases cardiomyocyte mitochondrial content. (A) After treated with QLQX at $0.5 \mu \mathrm{g} / \mathrm{mL}$ or control for 48 hours, H9C2 cells were stained with Mitotracker (green) and DAPI (blue), and then analyzed for mitochondrial size and fluorescence intensity using confocal microscope from 15 randomly selected images taken under 400x magnification $(\mathrm{n}=$ 4). Scale bar $=50$ um. (B) H9C2 cells treated with QLQX at $0.5 \mu \mathrm{g} / \mathrm{mL}$ for 48 hours were stained with Mitotracker and fluorescence of $\mathrm{GFP}^{+}$cells were quantified with flow cytometry $(\mathrm{n}=$ 6). (C) Mitochondria ultrastructures were visualized

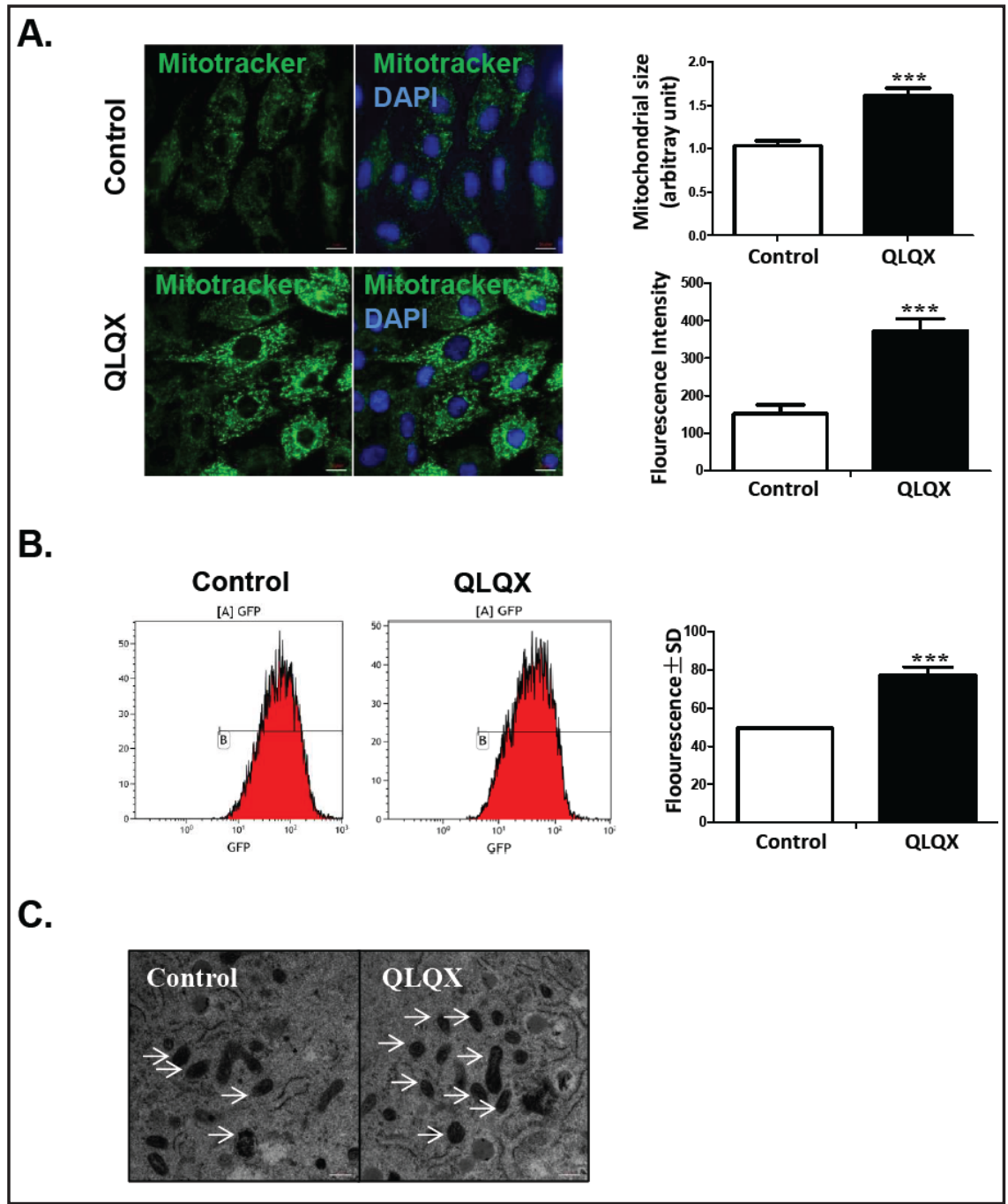
by transmission electron microscope in H9C2 cells treated with QLQX at $0.5 \mu \mathrm{g} / \mathrm{mL}$ for 48 hours $(\mathrm{n}=3)$. Original magnification: $9600 \mathrm{x}$. Scale bar $=2 \mu \mathrm{m}$. ${ }^{*}, P<0.05$; ${ }^{* *}, P<0.01$; ${ }^{* *}, P<0.001$.

1C). To determine whether the increased basal OCR or peak OCR was attributed to oxidative phosphorylation, we further analyzed mitochondrial uncoupling efficiency, which was demonstrated to be elevated with $0.5 \mu \mathrm{g} / \mathrm{mL}$ of QLQX treatment at 48 hours (Fig. 1D).

Knowing that cells consume the most oxygen at oxidative phosphorylation or glycolysis stage [19], we further detected the effects of QLQX on glycolysis. Extracellular acidification rate (ECAR), an indirect marker of lactate synthesis, was measured following $0.5 \mu \mathrm{g} / \mathrm{mL}$ of QLQX treatment from 6 to 48 hours. QLQX treatment at $0.5 \mu \mathrm{g} / \mathrm{mL}$ for 48 hours reduced both basal glycolysis and peak glycolysis (Fig. 2A and B). Interestingly, basal glycolysis and peak glycolysis were both enhanced with $0.5 \mu \mathrm{g} / \mathrm{mL}$ of QLQX compared with control treatment at earlier time points $(6,12$, and 24 hour) (Fig. 2A and B). Metabolic reliance, represented by the rate of basal oxidative metabolism to glycolytic metabolism, was significantly increased by $23.4 \%$ at $0.5 \mu \mathrm{g} / \mathrm{mL}$ of QLQX treatment for 48 hours, while reduced at earlier time points (6, 12, and 24 hour) (Fig. 2C). As cells were cultured in high-glucose media, we speculate that the reduced ECAR and elevated OCR observed at 48 hours might be related to enhanced oxygen consumption of $\mathrm{H} 9 \mathrm{C} 2$ cells after long duration of QLQX treatment. 


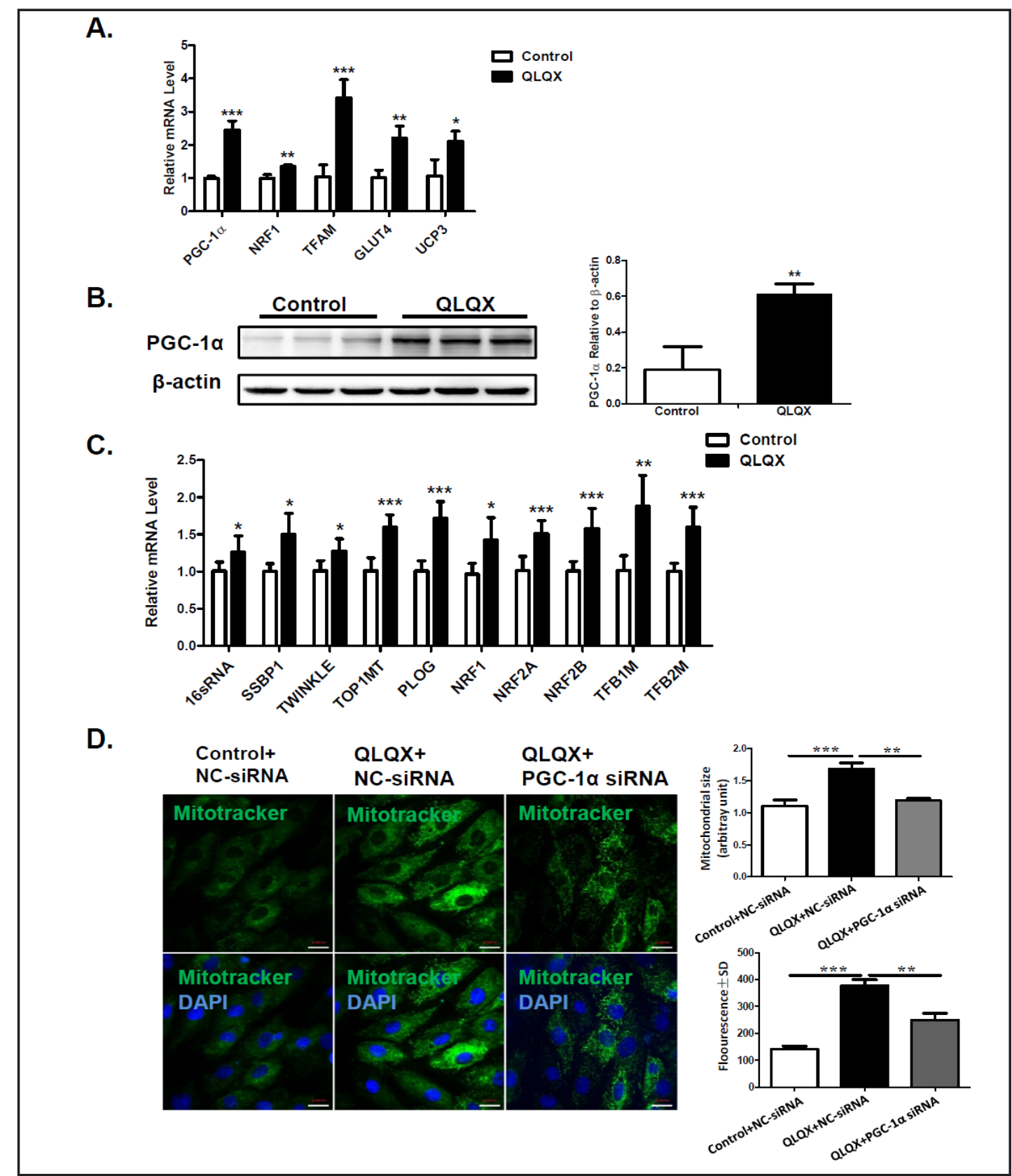

Fig. 4. QLQX upregulates mitochondrial biogenesis-related genes with PGC-1 $\alpha$ activation. (A) The expression levels of PGC- $1 \alpha$, NRF1, TFAM, GLUT4 and UCP3 were quantified by qRT-PCR following treatment of H9C2 cells with QLQX at $0.5 \mu \mathrm{g} / \mathrm{mL}$ for 48 hours $(\mathrm{n}=6)$. (B) Western blot and quantitative analysis of protein level of PGC- $1 \alpha$ in H9C2 cells following treatment with QLQX at $0.5 \mu \mathrm{g} / \mathrm{mL}$ for 48 hours $(\mathrm{n}=3)$. (C) Mitochondrial biogenesis-related genes measured by qRT-PCR following treatment of H9C2 cells with QLQX at $0.5 \mu \mathrm{g} / \mathrm{mL}$ for 48 hours $(n=6)$. (D) H9C2 cells were treated with QLQX at $0.5 \mu \mathrm{g} / \mathrm{mL}$ for 48 hours, with or without PGC$1 \alpha$ silencing. Mitotracker (green) staining of H9C2 cells were examined under confocal microscope at $400 \mathrm{x}$ magnification and analyzed for mitochondrial size and fluorescence intensity. Nuclei were counter stained with DAPI (blue) $(\mathrm{n}=4)$. ${ }^{*}, P<0.05$; **, $P<0.01$; ***, $P<0.001$.

\section{QLQX increases cardiomyocyte mitochondrial content}

Next, we investigated whether the increase in oxidative phosphorylation by QLQX was due to increased mitochondrial content by using Mitotracker staining, flow cytometry, and confocal microscopy. We found that cardiomyocyte mitochondrial content was significantly 


\section{Cellular Physiology Cell Physiol Biochem 2015;37:2246-2256 \\ \begin{tabular}{ll|l} 
DOI: 10.1159/000438580 & $\begin{array}{l}\text { O 2015 The Author(s). Published by S. Karger AG, Basel } \\
\text { www.karger.com/cpb }\end{array}$
\end{tabular} \\ Lin et al.: Metabolic Effects of Qiliqiangxin on Cardiomyocytes}

increased by QLQX treatment at $0.5 \mu \mathrm{g} / \mathrm{mL}$ for 48 hours, as determined by mitotracker green fluorescence intensity and mitochondrial size (Fig. 3A). We also performed flowmeter analysis to detect $\mathrm{GFP}^{+}$cells by Mitotracker staining and found that QLQX treatment increased mitochondrial content by $56.89 \%$ at a concentration of $0.5 \mu \mathrm{g} / \mathrm{mL}$ for 48 hours (Fig. 3B). To further confirm the effects of QLQX on mitochondria, we carried out transmission electron microscopy to visualize mitochondrial ultrastructures. Consistent to the results of confocal image and flowmeter analysis, QLQX treatment improved mitochondrial ultrastructure following 48 hours treatment of $0.5 \mu \mathrm{g} / \mathrm{mL}$ QLQX (Fig. 3C). These data suggested that QLQX treatment effectively elevated mitochondrial content and improved mitochondrial ultrastructure in cardiomyocytes.

\section{QLQX upregulates mitochondrial biogenesis with PGC-1 $\alpha$ activation}

To determine the molecular mechanisms responsible for enhanced mitochondrial content by QLQX treatment, we examined mitochondrial biogenesis-related gene expressions by quantitative real-time PCRs. As PGC- $1 \alpha$ has been described in previous studies to play a key role in regulating mitochondrial biogenesis, treatment with QLQX at $0.5 \mu \mathrm{g} / \mathrm{mL}$ for 48 hours caused a significant increase in PGC- $1 \alpha$ expression at mRNA level (Fig. 4A). Western blot analysis further confirmed the regulatory effect of QLQX on PGC-1 $\alpha$ (Fig. 4B). NRF1, a downstream target of PGC- $1 \alpha$, was also increased in $0.5 \mu \mathrm{g} / \mathrm{mL}$ QLQX-treated cardiomyocytes at 48 hours (Fig. 4A). TFAM, a downstream of NRF1 and a regulator of mitochondrial DNA expression, shared a similar change with NRF1 (Fig. 4A). Other important controllers of mitochondrial biogenesis, such as glucose transporter type 4 (GLUT4) and mitochondrial uncoupling protein 3 (UCP3), were also induced following treatment of $0.5 \mu \mathrm{g} / \mathrm{mL}$ QLQX for 48 hours (Fig. 4A). Meanwhile, we also examined other genes involved in energetic and mitochondrial biogenesis, such as mitochondrial DNA encoded genes and genes involved in mitochondrial DNA replication (16sRNA, SSBP1, TWINKLE, TOP1MT and PLOG), as well as genes involved in NRF family. QLQX-treated cardiomyocytes displayed significant increase in all of these genes at a concentration of $0.5 \mu \mathrm{g} / \mathrm{mL}$ for 48 hours (Fig. 4C). These results suggested that QLQX treatment enhanced mitochondrial content through upregulation of mitochondrial biogenesis-related genes with an activation of PGC- $1 \alpha$. Furthermore, we found that QLQX-induced enhancement of mitochondrial content in H9C2 cells could be significantly abolished by PGC- $1 \alpha$ siRNA (Fig. 4D), indicating that PGC- $1 \alpha$ activation is required for increased mitochondrial content induced by QLQX treatment in cardiomyocytes.

\section{Discussion}

Mitochondria are important for cardiomyocyte survival and maintenance of normal cardiac function [20]. Emerging evidence suggest that mitochondrial dysfunction may lead to the genesis of heart failure, which indicates a close association between mitochondrial biogenesis and cardiac function [21-23]. We previously reported the protective effects of traditional Chinese medicine QLQX on cardiomyocyte energy metabolism in mice with acute myocardial infarction or cardiac ischemia-reperfusion injury $[2,24]$. To better understand the effect of QLQX on physiological mitochondrial metabolism, we treated H9C2 cells with QLQX and examined oxidative metabolism/glycolysis by XF96 analyzer. Our work illustrated that QLQX $(0.5 \mu \mathrm{g} / \mathrm{mL})$ enhanced H9C2 cell glycolytic metabolism at earlier time points $(6$, 12 , and 24 hours), while enhanced $\mathrm{H} 9 \mathrm{C} 2$ cell oxidative metabolism at late time point (48 hours). Specifically, the data of XF96 analyzer demonstrated that H9C2 cells, which were cultured in high-glucose media and treated with QLQX, initially (6 to 24 hours treatment of QLQX) relied on glycolysis with corresponding lactate export and increased extracellular acidification. Thus, we hypothesized that the observed increase in cardiomyocyte glycolysis during early time points of QLQX treatment seemed to precede increased mitochondrial metabolism. In this case, $\mathrm{H} 9 \mathrm{C} 2$ cells were forced to utilize glycolysis to meet energy requirements until mitochondrial content is increased enough to meet energy needs through oxidative metabolism. 


\section{Cellular Physiology Cell Physiol Biochem 2015;37:2246-2256 and Biochemistry Published online: November 29, 2015 lo $\begin{aligned} & \text { C } 2015 \text { The Author(s). Published by S. Karger AG, Basel } \\ & \text { www.karger.com/cpb }\end{aligned}$ \\ Lin et al.: Metabolic Effects of Qiliqiangxin on Cardiomyocytes}

After longer duration of QLQX treatment for 48 hours, H9C2 cells preferentially utilized oxidative means and exhibited decreased acidification and increased oxygen consumption, which suggested that complete carbohydrate oxidation was occurring at a higher rate simultaneously with an increase in mitochondrial content and upregulation of mitochondrial biogenesis-related genes, including 16sRNA, SSBP1, TWINKLE, TOP1MT and PLOG. Therefore, long term treatment of QLQX could substantially increase cardiomyocyte oxidative metabolism under basal condition and lead to increased peak metabolic potential during cell stress.

PGC- $1 \alpha$, a coactivator of PPAR $\gamma$, serves as an inducible booster involved in mitochondrial biogenesis $[25,26]$. In the current study, PGC- $1 \alpha$ as well as its downstream effectors, including NRF1, TFAM and other related genes involved in NRF family was also found to be upregulated in cardiomyocytes after QLQX treatment at $0.5 \mu \mathrm{g} / \mathrm{mL}$ for 48 hours. Furthermore, silencing PGC- $1 \alpha$ could totally abolish the increase in mitochondrial content induced with QLQX treatment. These results provide evidence that PGC- $1 \alpha$ is required for QLQX-associated enhancement in cardiomyocyte mitochondrial energy metabolism.

Several limitations of the present study should be highlighted. First, the effect of QLQX on mitochondrial biogenesis was explored in cardiomyocytes during basal condition, it deserves to further evaluate whether and how QLQX could perform protective effect on cardiomyocyte mitochondrial metabolism under pathological condition. Second, the molecular mechanisms responsible for the protective effect of QLQX on mitochondrial biogenesis remain yet to be elucidated. Third, further experiments are needed to reinforce the conclusion that QLQX enhances cardiomyocyte metabolism by using primary culture of cardiomyocytes, for example neonatal rat cardiomyocytes.

In conclusion, the present study demonstrates that QLQX improves cardiomyocyte metabolism and increases mitochondrial biogenesis via activation of PGC- $1 \alpha$, which might contribute to its clinical therapeutic effects on adverse cardiac remodeling and heart failure.

\section{Acknowledgments}

This work was supported by the grants from National Natural Science Foundation of China (81370332 and 81170201 to XL Li, 81570362 and 81200169 to JJ Xiao, 81400647 to Y Bei), the Priority Academic Program Development of Jiangsu Higher Education Institutions (PAPD20102013 to XL Li), Innovation Program of Shanghai Municipal Education Commission (13YZ014 to JJ Xiao), Foundation for University Young Teachers by Shanghai Municipal Education Commission (year 2012, to JJ Xiao), Innovation fund from Shanghai University (sdcx2012038 to JJ Xiao), and Program for the integration of production, teaching and research for University Teachers supported by Shanghai Municipal Education Commission (year 2014, to JJ Xiao). Dr XL Li is an Associate Fellow at the Collaborative Innovation Center for Cardiovascular Disease Translational Medicine.

\section{Disclosure Statement}

Dr. Xinli Li and Junjie Xiao received research grants from Shijiazhuang Yiling Pharmaceutical Co., Ltd. All other authors have reported that they have no relationships to disclose.

\section{References}

1 Li X, Zhang J, Huang J, Ma A, Yang J, Li W, Wu Z, Yao C, Zhang Y, Yao W, Zhang B, Gao R, Efficacy, Safety of Qili Qiangxin Capsules for Chronic Heart Failure Study G: A multicenter, randomized, double-blind, parallelgroup, placebo-controlled study of the effects of qili qiangxin capsules in patients with chronic heart failure. J Am Coll Cardiol 2013;62:1065-1072. 


\section{Cellular Physiology Cell Physiol Biochem 2015;37:2246-2256

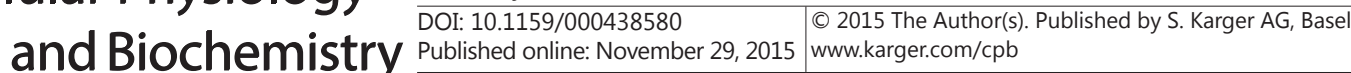 \\ Lin et al.: Metabolic Effects of Qiliqiangxin on Cardiomyocytes}

2 Tao L, Shen S, Fu S, Fang H, Wang X, Das S, Sluijter JP, Rosenzweig A, Zhou Y, Kong X, Xiao J, Li X: Traditional chinese medication qiliqiangxin attenuates cardiac remodeling after acute myocardial infarction in mice. Sci Rep 2015;5:8374.

3 Zhang J, Wei C, Wang H, Tang S, Jia Z, Wang L, Xu D, Wu Y: Protective effect of qiliqiangxin capsule on energy metabolism and myocardial mitochondria in pressure overload heart failure rats. Evid Based Complement Alternat Med 2013;2013:378298.

4 Chen F, Wu JL, Fu GS, Mou Y, Hu SJ: Chronic treatment with qiliqiangxin ameliorates aortic endothelial cell dysfunction in diabetic rats. J Cardiovasc Pharmacol Ther 2015;20:230-240.

5 Warda M, Kim HK, Kim N, Ko KS, Rhee BD, Han J: A matter of life, death and diseases: Mitochondria from a proteomic perspective. Expert Rev Proteomics 2013;10:97-111.

6 Huang X, Sun L, Ji S, Zhao T, Zhang W, Xu J, Zhang J, Wang Y, Wang X, Franzini-Armstrong C, Zheng M, Cheng $\mathrm{H}$ : Kissing and nanotunneling mediate intermitochondrial communication in the heart. Proc Natl Acad Sci U S A 2013;110:2846-2851.

7 Handy DE, Loscalzo J: Redox regulation of mitochondrial function. Antioxid Redox Signal 2012;16:13231367.

8 Park KS, Wiederkehr A, Wollheim CB: Defective mitochondrial function and motility due to mitofusin 1 overexpression in insulin secreting cells. Korean J Physiol Pharmacol 2012;16:71-77.

9 Senese R, Valli V, Moreno M, Lombardi A, Busiello RA, Cioffi F, Silvestri E, Goglia F, Lanni A, de Lange P: Uncoupling protein 3 expression levels influence insulin sensitivity, fatty acid oxidation, and related signaling pathways. Pflugers Arch 2011;461:153-164.

10 Lauritzen KH, Kleppa L, Aronsen JM, Eide L, Carlsen H, Haugen OP, Sjaastad I, Klungland A, Rasmussen LJ, Attramadal H, Storm-Mathisen J, Bergersen LH: Impaired dynamics and function of mitochondria caused by mtdna toxicity leads to heart failure. Am J Physiol Heart Circ Physiol 2015;309:H434-449.

11 Shoag J, Arany Z: Regulation of hypoxia-inducible genes by pgc-1 alpha. Arterioscler Thromb Vascu Biol 2010;30:662-666.

12 Lukaszuk B, Miklosz A, Chabowski A, Górski J: Modest decrease in PGC1 $\alpha$ results in TAG accumulation but not in insulin resistance in L6 myotubes. Cell Physiol Biochem 2015;35:1609-1622.

13 Johnson RF, Witzel, II, Perkins ND: P53-dependent regulation of mitochondrial energy production by the rela subunit of nf-kappab. Cancer Res 2011;71:5588-5597.

14 Lin J, Wu PH, Tarr PT, Lindenberg KS, St-Pierre J, Zhang CY, Mootha VK, Jager S, Vianna CR, Reznick RM, Cui L, Manieri M, Donovan MX, Wu Z, Cooper MP, Fan MC, Rohas LM, Zavacki AM, Cinti S, Shulman GI, Lowell BB, Krainc D, Spiegelman BM:Defects in adaptive energy metabolism with cns-linked hyperactivity in pgc1alpha null mice. Cell 2004;119:121-135.

15 Leone TC, Lehman JJ, Finck BN, Schaeffer PJ, Wende AR, Boudina S, Courtois M, Wozniak DF, Sambandam N, Bernal-Mizrachi C, Chen Z, Holloszy JO, Medeiros DM, Schmidt RE, Saffitz JE, Abel ED, Semenkovich CF, Kelly DP: Pgc-1alpha deficiency causes multi-system energy metabolic derangements: Muscle dysfunction, abnormal weight control and hepatic steatosis. PLoS Biol 2005;3:e101.

16 Lehman JJ, Barger PM, Kovacs A, Saffitz JE, Medeiros DM, Kelly DP: Peroxisome proliferator-activated receptor gamma coactivator-1 promotes cardiac mitochondrial biogenesis. J Clin Invest 2000;106:847-856.

17 Schreiber SN, Knutti D, Brogli K, Uhlmann T, Kralli A: The transcriptional coactivator pgc-1 regulates the expression and activity of the orphan nuclear receptor estrogen-related receptor alpha (erralpha). J Biol Chem 2003;278:9013-9018.

18 May-Panloup P, Vignon X, Chretien MF, Heyman Y, Tamassia M, Malthiery Y, Reynier P: Increase of mitochondrial DNA content and transcripts in early bovine embryogenesis associated with upregulation of mttfa and nrf1 transcription factors. Reprod Biol Endocrinol 2005;3:65.

19 Vaughan RA, Gannon NP, Barberena MA, Garcia-Smith R, Bisoffi M, Mermier CM, Conn CA, Trujillo KA: Characterization of the metabolic effects of irisin on skeletal muscle in vitro. Diabetes Obes Meta 2014;16:711-718.

20 Hammerling BC, Gustafsson AB: Mitochondrial quality control in the myocardium: Cooperation between protein degradation and mitophagy. J Mol Cell Cardiol 2014;75:122-130.

21 Aubert G, Vega RB, Kelly DP: Perturbations in the gene regulatory pathways controlling mitochondrial energy production in the failing heart. Biochim Biophys Acta 2013;1833:840-847. 
22 Wang H, Bei Y, Lu Y, Sun W, Liu Q Wang Y, Cao Y, Chen P, Xiao J, Kong X: Exercise prevents cardiac injury and improves mitochondrial biogenesis in advanced diabetic cardiomyopathy with PGC-1 $\alpha$ and Akt activation. Cell Physiol Biochem 2015;35:2159-2168.

23 Tao L, Bei Y, Lin S, Zhang H, Zhou Y, Jiang J, Chen P, Shen S, Xiao J, Li X: Exercise training protects against acute myocardial infarction via improving myocardial energy metabolism and mitochondrial biogenesis. Cell Physiol Biochem 2015;37:162-175.

24 Zhou Y, Fang H, Lin S, Shen S, Tao L, Xiao J, Li X: Qiliqiangxin protects against cardiac ischemia-reperfusion injury via activation of the mTOR pathway. Cell Physiol Biochem 2015;37:454-464.

25 Zhang P, Lu Y, Yu D, Zhang D, Hu W: TRAP1 provides protection against myocardial ischemia-reperfusion injury by ameliorating mitochondrial dysfunction. Cell Physiol Biochem 2015;36:2072-2082.

26 Chan S, Chan GC, Ye J, Lian Q, Chen J, Yang M: Thrombopoietin protects cardiomyocytes from iron-overload induced oxidative stress and mitochondrial injury. Cell Physiol Biochem 2015;36:2063-2071. 